Revisão / Review

\title{
Identificação e caracterização de variantes novas e raras da hemoglobina humana
}

\section{Identification of characterization of novel and rare variants of human hemoglobin}

\author{
Elza M. Kimura ${ }^{1}$ \\ Denise M. Oliveiral \\ Susan E. D. C. Jorgel \\ Cristina F. Abreu ${ }^{1}$ \\ Dulcinéia M. Albuquerque ${ }^{2}$ \\ Fernando F. Costa ${ }^{2}$ \\ Maria de Fátima Sonati ${ }^{1}$
}

\begin{abstract}
As anormalidades estruturais da hemoglobina estão entre as doenças genéticas mais comumente encontradas nas populações humanas. O Laboratório de Hemoglobinopatias do Departamento de Patologia Clinica da Faculdade de Ciências Médicas da Universidade Estadual de Campinas - Unicamp, localizado em Campinas, no estado de São Paulo, região Sudeste do Brasil, realizou, em seus 27 anos de existência, cerca de 130.000 diagnósticos. Entre as variantes estruturais detectadas, as hemoglobinas $S$, C e D-Punjab foram, como esperado, as mais freqüentes, porém um número expressivo de outras hemoglobinas anômalas, novas e raras, também foi encontrado. Esses achados estão sumarizados no presente artigo. Rev. bras. hematol. hemoter. 2008;30(4):316-319.
\end{abstract}

Palavras-chave: Hemoglobinopatias hereditárias; variantes estruturais; genes de globinas; população brasileira.

\section{Introdução}

Mutações nos genes das globinas podem levar à produção de hemoglobinas estruturalmente alteradas. ${ }^{1}$ Há, atualmente, mais de 900 variantes estruturais descritas, a maioria ocasionada por simples substituições de bases no DNA, com a correspondente troca de aminoácidos na proteína. ${ }^{2}$ Embora a maior parte dos casos seja de cadeias $\beta$, alterações de cadeias $\alpha, \gamma$ e $\delta$ são também relativamente comuns. Há um grande contingente não relacionado à sintomatologia clínica, mas algumas alterações afetam a estabilidade e/ou solubilidade da molécula ou modificam suas propriedades funcionais, levando às anemias hemolíticas e às eritrocitoses $\mathrm{e}$ cianoses, respectivamente. Há ainda variantes alongadas ou extremamente instáveis que resultam em fenótipos talassêmicos. Entre as variantes clinicamente importantes, a $\mathrm{Hb} \mathrm{S}$ $\left(\alpha_{2} \beta_{2}^{\mathrm{s}}\right)$, da anemia falciforme, é sem dúvida a mais conhecida. ${ }^{1,2}$

O Laboratório de Hemoglobinopatias do Departamento de Patologia Clínica da Faculdade de Ciências Médicas da
Unicamp, desde sua implantação, em 1980, analisou cerca de 130.000 amostras de sangue, provenientes de indivíduos com suspeita clínica de hemoglobinopatias e seus familiares, de doadores de sangue nos quais se detectou a presença de hemoglobinas estruturalmente alteradas, de programas de triagem neonatal e, ainda, de um programa sistemático de triagem de variantes desenvolvido no próprio laboratório. A maior parte das amostras é oriunda de indivíduos da região sudeste, mas outras regiões brasileiras também contribuíram para essa casuística, como a região nordeste e a região sul, bem como alguns outros países da América Latina, como a Argentina, o México, o Uruguai e a Venezuela. Até o momento, e excluindo-se as alterações de cadeias $\delta$, genericamente intituladas $\mathrm{HbA}_{2}, 10$ variantes novas e 51 variantes raras foram detectadas e identificadas, sendo 9 delas mutações de novo; 21 são alterações de cadeias $\alpha, 31$ de cadeias $\beta$ e 9 de cadeias $\gamma$, entre $\gamma^{G}$ e $\gamma^{A}$. A maioria das variantes já descritas está sendo detectada no Brasil pela primeira vez. As tabelas de 1 a 4 apresentam esses resultados.

\footnotetext{
${ }^{I}$ Departamento de Patologia Clínica, Faculdade de Ciências Médicas, Universidade Estadual de Campinas - Unicamp, Campinas-SP. ${ }^{2}$ Centro de Hematologia e Hemoterapia, Unicamp, Campinas, SP. 
Tabela 1. Variantes Novas de $\mathrm{Hb}$

\begin{tabular}{lc}
\hline Hemoglobina & Mutação \\
Hb Fetal-Campinas & GamaA 121(GH4) Glu>Gln \\
Hb Fetal-Joanópolis & GamaG 73 (E17) Asp>Ala \\
Hb Fetal-Paulínia & GamaG 80 (EF4) Asp>Tir \\
Hb Boa Esperança & Alfa2 16(A14) Lis>Tre \\
Hb Campinas & Alfa2 26(B7) Ala>Val \\
Hb Bom Jesus da Lapa & Alfa1 30(B11) Glu>Ala \\
Hb Itapira & Alfa1 30(B11) Glu>Val \\
Hb Rio Claro & Beta 33(B15) Val>Met \\
Hb Poços de Caldas & Beta 61(E5) Lis>Gln \\
Hb Florida* & Beta 141 (-C); seqüência C- terminal \\
& modificada: (141)Tri-Pro-Tre-Ser-Ile- \\
& Tre-Lis-Leu-Ala-Fen-Leu-Leu-Ser-Asn-- \\
& Fen-(156)Tir-COOH \\
\hline
\end{tabular}

*Mutação de novo

Tabela 2. Variantes raras de Cadeias $\alpha$

\begin{tabular}{|c|c|}
\hline Hemoglobina & Mutação \\
\hline Hb Kurosaki* & Alfa1 7(A5) Lis >Glu \\
\hline $\mathrm{Hb}$ J-Paris & Alfa2 12(A10) Ala>Asp \\
\hline $\mathrm{Hb}$ Hasharon & $\begin{array}{c}\text { Híbrido Alfa2/ Alfa1 47(CE5) } \\
\text { Asp>His }\left(\alpha^{3.7}\right)\end{array}$ \\
\hline $\mathrm{Hb}$ J-Rovigo & Alfa2 53(E2) Ala>Asp \\
\hline Hb Shaare Zedek & Alfa2 56(E5) Lis>Glu \\
\hline $\mathrm{Hb}$ Pontoise & Alfa2 63(E12) Ala>Asp \\
\hline Hb Ube-2 & Alfa1 68(E17) Asn>Asp \\
\hline Hb Daneshgah-Tehran & Alfa2 72(EF1) His>Arg \\
\hline Hb G-Pest* & Alfa2 74(EF3) Asp>Asn \\
\hline Hb Stanleville-II & $\begin{array}{c}\text { Hibrido Alfa2/ Alfa1 78(EF7) } \\
\text { Asn>Lis }\left(\alpha^{3.7}\right)\end{array}$ \\
\hline Hb Tamano & Alfa1 89(FG1) His>Arg \\
\hline $\mathrm{Hb}$ Cemenelum & Alfa2 92(FG4) Arg>Tri \\
\hline Hb Setif & Alfa2 94(G1) Asp>Tir \\
\hline Hb Sunshine Seth* & Alfa1 94(G1) Asp>His \\
\hline Hb Westmead* & Alfa2 122(H5) His $>$ Gln \\
\hline Hb Jackson & Alfa2 $127(\mathrm{H} 10)$ Lis $>$ Asn \\
\hline Hb Icaria & $\begin{array}{c}\text { Alfa2 142, Stop>Lis; seqüência C- terminal } \\
\text { modificada: (142)Lis-Ala-Gli-Ala-Ser-Val-Ala- } \\
\text { Val-Pro-Pro-Ala-Arg-Tri-Ala-Ser-Gln-Arg-Ala- } \\
\text { Leu-Leu-Pro- Ser-Leu-His-Arg-Pro-Fen-Leu- } \\
\text { Val-Fen-(172)Glu-COOH }\end{array}$ \\
\hline
\end{tabular}

A principal forma de detecção é feita a partir da mudança na carga elétrica da variante em função da troca de aminoácidos ocorrida. A Figura 1 resume a estratégia metodológica utilizada. A eletroforese de hemoglobinas em acetato de celulose, em pH alcalino, e a cromatografia líquida de alta performance (HPLC - High Performance Liquid Chromatography), de troca catiônica, são as técnicas empregadas na triagem, esta última permitindo também a quantificação da fração anômala e das hemoglobinas $\mathrm{A}_{2}$ e Fetal. As variantes assim detectadas são então submetidas à eletroforese em gel de ágar, em $\mathrm{pH}$ ácido, aos testes de solubilidade e estabilidade (térmica, em n-butanol e em isopropanol) e
Tabela 3. Variantes Raras de Cadeias $\beta$

\begin{tabular}{|c|c|}
\hline Hemoglobina & Mutação \\
\hline $\mathrm{Hb}$ Deer Lodge & Beta 2(NA2) His>Arg \\
\hline Hb G-San José & Beta 7(A4) Glu>Gli \\
\hline $\mathrm{Hb}$ Porto Alegre & Beta 9(A6) Ser>Cis \\
\hline $\mathrm{Hb} \mathrm{J}$-Baltimore & Beta 16(A13) Gli>Asp \\
\hline $\mathrm{Hb}$ Miyashiro & Beta 23(B5) Val>Gli \\
\hline $\mathrm{Hb} \mathrm{E}$ & Beta 26(B8) Glu>Lis \\
\hline $\mathrm{Hb}$ Grange-Blanche & Beta 27(B9) Ala>Val \\
\hline $\mathrm{Hb}$ Pitie-Salpetriere & Beta 34(B16) Val>Fen \\
\hline Hb Hammersmith* & Beta 42 (CD1) Fen>Ser \\
\hline Hb Hoshida & Beta 43(CD2) Glu>Gln \\
\hline $\mathrm{Hb}$ Osu Christiansborg* & Beta 52(D3) Asp>Asn \\
\hline $\mathrm{Hb}$ Dhofar & Beta 58(E2) Pro>Arg \\
\hline $\mathrm{Hb}$ Zurich & Beta 63(E7) His>Arg \\
\hline Hb Korle-Bu & Beta 73(E17) Asp >Asn \\
\hline Hb Santa Ana* & Beta 88(F4) Leu>Pro \\
\hline Hb Redondo* & Beta 92(F8) His>Asn \\
\hline $\mathrm{Hb}$ N-Baltimore & Beta 95(FG2) Lis>Glu \\
\hline Hb Köln & Beta 98(FG5) Val>Met \\
\hline $\mathrm{Hb}$ Coimbra & Beta 99(G1) Asp > Glu \\
\hline Hb Camperdown & Beta 104(G6) Arg>Ser \\
\hline $\mathrm{Hb}$ Indianápolis & Beta 112(G14) Cis>Arg \\
\hline Hb Miami & Beta 116(G18) His>Pro \\
\hline Hb D-Punjab & Beta $121(\mathrm{GH} 4)$ Glu>Gln \\
\hline $\mathrm{Hb} \mathrm{Hofu}$ & Beta $126(\mathrm{H} 4)$ Val>Glu \\
\hline Hb J-Guantanamo & Beta 128(H6) Ala>Asp \\
\hline $\mathrm{Hb}$ K-Woolwich & Beta 132(H10) Lis $>$ Gln \\
\hline Hb Lepore-Baltimore & $\begin{array}{c}\text { Híbrido Delta-Beta } \\
\text { (delta } 50 \text { Ser; beta } 86 \mathrm{Ala} \text { ) }\end{array}$ \\
\hline $\begin{array}{l}\mathrm{Hb} \text { Lepore- Boston- } \\
\text { Washington }\end{array}$ & $\begin{array}{c}\text { Híbrido Delta-Beta } \\
\text { (delta } 87 \mathrm{GIn} \text {; beta } 116 \text { IVS-II-8) }\end{array}$ \\
\hline
\end{tabular}

Tabela 4. Variantes raras de Cadeias $\gamma$

$\begin{array}{cc}\text { Hemoglobina } & \text { Mutação } \\ \text { Hb F-Pendergras } & \text { GamaA 36(C2) Pro>Arg } \\ \text { Hb F-Yamaguchi } & \text { GamaA 80(EF4) Asp>Asn E GamaA } \\ \text { Hb F-Dickinson } & \text { 75(E19) lle>Tir } \\ \text { Hb F-Urumqi } & \text { GamaA 97(FG4) His>Arg } \\ \text { Hb F-Carlton } & \text { GamaG 121(G4) Asp }>\text { Gli } \\ \text { Hb F-Port Royal } & \text { GamaG 125(H3) Glu>Ala }\end{array}$

à análise de cadeias globínicas, feita anteriormente por eletroforese e atualmente por HPLC de fase reversa (RPHPLC). Paralelamente, os eritrócitos, após análise por contador eletrônico, são investigados quanto à presença de corpos de Heinz e de inclusão. ${ }^{3}$

Uma vez identificada a cadeia mutante, o gene correspondente é então seletivamente amplificado pela reação em cadeia da polimerase (PCR), e seqüenciado. ${ }^{4-6}$ A confirmação 


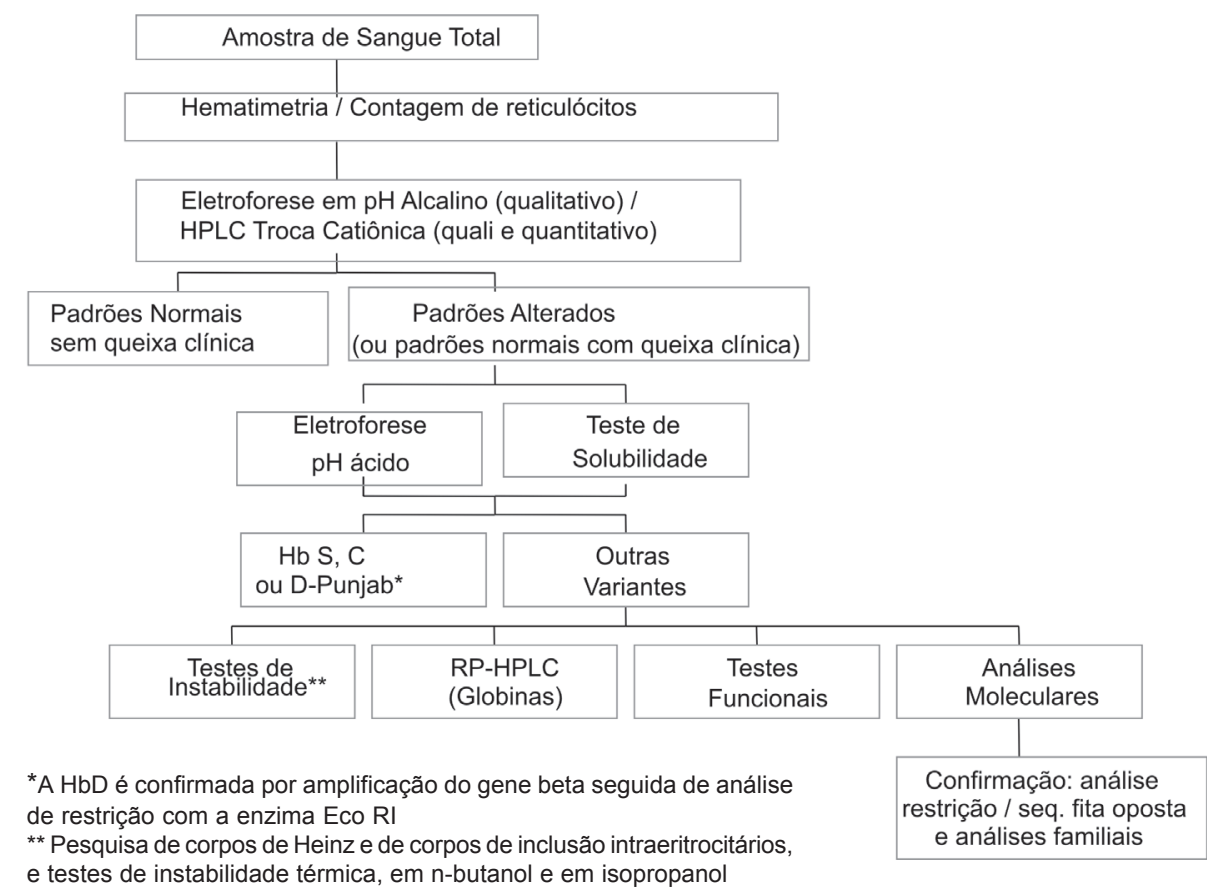

Figura 1. Representação Esquemática da Identificação e Caracterização de Variantes Estruturais $\mathrm{da} \mathrm{Hb}$

da mutação é feita, sempre que possível, por análise com enzimas de restrição e/ou seqüenciamento da fita oposta de DNA, além da análise familial. No caso de variantes estruturais de cadeias $\alpha$, o status numérico desses genes é ainda investigado. ${ }^{7,8}$

Finalmente, as propriedades funcionais da molécula anômala são avaliadas pelo método de cinética química de Rossi-Fanelli e Antonini, de 1958, através da curva de dissociação da hemoglobina com o $\mathrm{O} 2$, da determinação da cooperatividade entre as cadeias globínicas e do efeito Bohr, na ausência e na presença de fosfatos orgânicos. ${ }^{9}$

Dentre as variantes clinicamente importantes aqui encontradas estão as hemoglobinas Hammersmith, Zürich, Redondo, Köln, Indianápolis, Miami, Hb Sunshine Seth e Miyashiro, proteínas instáveis que resultam em anemia hemolítica crônica de intensidade moderada a grave, as hemoglobinas Lepore-Baltimore e Lepore-Boston, híbridos de cadeias $\delta$ e $\beta$ associados a fenótipo talassêmico $\left(\delta \beta^{+}\right)$, a Hb Florida, constituída de cadeias $\beta$ com 156 aminoácidos, hiperinstável e com fenótipo dominante de talassemia intermediária, a $\mathrm{Hb} \mathrm{E}$, também com fenótipo $\beta$-talassêmico, a $\mathrm{Hb}$ Coimbra e a Pitie-Salpetriere, encontradas em pacientes com eritrocitose, e as variantes Hasharon e Stanleyville-II, sempre associadas à deleção $-\alpha^{3,7}$ e ao correspondente fenótipo $\alpha$-talassêmico. ${ }^{10-17}$

A Hb Boa Esperança foi detectada em dois portadores assintomáticos (doadores de sangue), mas os estudos in vitro revelaram propriedades funcionais alteradas, compensadas in vivo, provavelmente, pela maior proporção de hemoglobina normal, de $75 \% .{ }^{18}$ Já a $\mathrm{Hb}$ Itapira foi a variante representada em menor porcentagem, com cerca de $5,5 \%$, porque a mutação que a deu origem ocorreu em um alelo $\alpha$ triplicado $\left(\alpha \alpha \alpha^{\text {anti-3,7,7}}\right) .^{18}$

Algumas associações incomuns foram observadas. Heterozigotos da $\mathrm{Hb}$ Porto Alegre são geralmente assintomáticos, mas sua concomitância com a Hb Santa Ana, gerada por mutação de novo, levou a um quadro clínico de anemia hemolítica grave, com necessidade de esplenectomia. ${ }^{19} \mathrm{~A} \mathrm{Hb}$ Icaria, uma variante alongada e instável de cadeias $\alpha$, foi recentemente encontrada em associação com a deleção $-(\alpha)^{20,5}$, resultando em Doença da $\mathrm{Hb} \mathrm{H}$ com altos níveis das hemoglobinas $\mathrm{H} \mathrm{e}$ Bart's, de $15 \%$ e $19 \%$, respectivamente. A Hb Miami foi detectada em uma criança heterozigota da talassemia $\beta^{+}$, o que levou a um quadro de anemia hemolítica bastante grave. Esta paciente havia sido primeiramente estudada por Hoyer et al., na Clínica Mayo, nos Estados Unidos. ${ }^{20} \mathrm{~A} \mathrm{Hb}$ $\mathrm{S}$, além das associações com as hemoglobinas $\mathrm{C}$ e D e com a talassemia $\beta$, foi detectada em interação com as variantes J-Rovigo, Grange-Blanche e Stanleyville-II. ${ }^{10,17}$ Esta última foi também detectada em associação com a Hb Campinas, uma das variantes novas descritas em nosso laboratório. A $\mathrm{Hb}$ Rio Claro, outra nova variante, foi encontrada em uma paciente também heterozigota da Hb Hasharon e, conseqüentemente, da talassemia $\alpha^{+} .{ }^{21}$ As hemoglobinas D e JRovigo foram ainda detectadas em concomitância à talassemia $\beta .^{10}$

Esses resultados ilustram a expressiva variabilidade de hemoglobinas presente nas populações. Do ponto de vista clínico, seu correto diagnóstico previne procedimentos e esquemas terapêuticos equivocados, particularmente quando os indivíduos apresentam microcitose e hipocromia, ou nos casos de variantes com o mesmo comportamento eletroforético ou cromatográfico daquelas mais freqüentemente observadas, como a Hb S. Do ponto de vista bioquímico, o estudo das variantes e de suas respectivas substituições de resíduos tem fornecido um importante modelo para a compreensão dos aspectos estruturais e funcionais desta e de outras proteínas, o mesmo ocorrendo com os genes de globinas e seus sistemas regulatórios. Do ponto de vista de genética de populações, sua investigação contribui de maneira significativa para o conhecimento da composição étnica e dos graus de miscigenação entre os diferentes grupos populacionais. 


\section{Abstract}

Hemoglobin structural abnormalities are among the most commonly found human genetic diseases. The Laboratory of Hemoglobinopathies in the Clinical Pathology Department of the Medical Sciences School of the State University in Campinas - Unicamp, São Paulo, Southeastern Brazil, carried out, in its 27 years of activity, about 130,000 diagnoses. As expected, hemoglobins $S, C$ and $D$ were the most frequently observed variants, but an expressive number of other abnormal, novel and rare hemoglobins, was also detected. These findings are summarized in the present article.. Rev. bras. hematol. hemoter. 2008;30(4):316-319.

Key words: Hereditary hemoglobinopathies; structural variants; globin genes; Brazilian population.

\section{Agradecimentos}

À Fundação de Amparo à Pesquisa do Estado de São Paulo (Fapesp), ao Conselho Nacional de Desenvolvimento Científico e Tecnológico $(\mathrm{CNPq})$ e à Coordenação de Aperfeiçoamento de Pessoal de Nivel Superior (Capes, pelo suporte financeiro aos projetos de pesquisa e pelas bolsas de estudo concedidas aos alunos de iniciação científica e de pós-graduação.

\section{Referências Bibliográficas}

1. Steinberg MH, Forget BG, Higgs DR, Nagel RL. Disorders of Hemoglobin. Cambridge University Press, New York, 2001.

2. Hardison RC, Chui DH, Giardine B et al. HbVar: A relational database of human hemoglobin variants and thalassemia mutations at the globin gene server. Hum Mutat. 2002;19(3):225-33 (http:// globin.cse.psu.edu).

3. Dacie JV, Lewis SM. Hematologia Prática de Dacie e Lewis, 9ª ed., Artmed, Porto Alegre, 2006.

4. Wenning MR, Kimura EM, Jorge SB et al. Molecular characterization of hemoglobins Kurosaki [alpha7 Lys-Glu], GPest [alpha74 Asp-Asn], Stanleyville-II [alpha78 Asn-Lys] and JRovigo [alpha53 Ala-Asp]. Acta Haematol. 1999; 102 (4):203-5.

5. Kimura EM, Jorge SB, Ogo SH et al. A novel beta-globin variant: Hb Poços de Caldas [beta 61(E5)Lys-Gln]. Hemoglobin. 2002; 26(4):385-8.

6. Duarte DF, Kimura EM, Albuquerque DM et al. Structural alterations of the gamma-globin genes in a Brazilian population. Hemoglobin. 2004;28(1):73-7.

7. Dodé C, Krishnamoorthy R, Lamb J et al. Rapid analysis of -alpha 3.7 thalassaemia and alpha alpha alpha anti 3.7 triplication by enzymatic amplification analysis. Br J Haematol. 1993;83 (1):105-11.

8. Tan AS, Quah TC, Low PS et al. A rapid and reliable 7-deletion multiplex polymerase chain reaction assay for alpha-thalassemia. Blood. 2001;98(1):250-1.

9. Rossi-Fanelli A, Antonini E. Studies on the oxygen and carbon monoxide equilibria of human myoglobin. Arch Biochem Biophys. 1958;77(2):478-92.

10. Abreu CF, Oliveira DM, Albuquerque DM et al. Hemoglobinas novas, raras e mutações de novo em pacientes atendidos no $\mathrm{HC}$ da Unicamp. Anais do $40^{\circ}$. Congresso Brasileiro de Patologia Clínica e Medicina Laboratorial (ISBN 85-60167-00-5), resumo 320, Curitiba (PR), setembro, 2006.
11. Sonati MF, Kimura EM, Abreu CF et al. Hemoglobin Hammersmith [beta42 (CD1) Phe-Ser] in a Brazilian girl with congenital Heinz body hemolytic anemia. Pediatr Blood Cancer. 2006;47(6):855-6.

12. Miranda SR, Kimura EM, Saad ST et al. Identification of Hb Zürich [alpha 2 beta 2(63)(E7)His-Arg] by DNA analysis in a Brazilian family. Hemoglobin. 1994;18(4-5):337-41.

13. Fattori A, Kimura EM, Albuquerque DM et al. Hb Indianapolis [beta112 (G14) Cys-Arg] as the probable cause of moderate hemolytic anemia and renal damage in a Brazilian patient. Am J Hematol. 2007;82(7):672-5

14. Miranda SR, Figueiredo MS, Kerbauy J et al. Hb Lepore Baltimore (delta 50Ser beta 86Ala) identified by DNA analysis in a Brazilian family. Acta Haematol. 1994;91(1):7-9.

15. Weinstein BI, Erramouspe B, Albuquerque DM et al. Hb Florida: a novel elongated $\mathrm{C}$-terminal beta-globin variant causing dominant beta-thalassemia phenotype. Am J Hematol. 2006;81(5):358-60.

16. Fattori A, Kimura EM, Albuquerque DM et al. Polycythemia and Hb Coimbra [beta99 (G1) Asp-Glu] in Brazil. Genet Mol Biol. 2006;29(2):200-2.

17. Costa FF, Sonati MF, Zago MA. Hemoglobin Stanleyville II (alpha 78 Asn-Lys) is associated with a $3.7-\mathrm{kb}$ alpha-globin gene deletion. Hum Genet. 1991;86(3):319-20.

18. Jorge SE, Kimura EM, Oliveira DM et al. Three new alpha-globin variants: Hb Itapira [alpha30(B11)Glu-->Val (alpha1)], Hb Bom Jesus Da Lapa [alpha30(B11)Glu-->Ala (alpha1)] and Hb Boa Esperança [alpha16(A14)Lys-->Thr (alpha2)]. Hemoglobin. 2007; 31(2):151-7

19. Gonçalves MS, Sonati MF, Kimura $\mathrm{M}$ et al. Association of $\mathrm{Hb}$ Santa Ana [alpha 2 beta (2)88(F4)Leu- $>$ Pro] and Hb Porto Alegre [alpha 2 beta (2)9(A6)Ser- > Cys] in a Brazilian female. Hemoglobin. 1994;18(3):235-9.

20. Hoyer JD, Baxter JK, Moran AM et al. Two unstable beta chain variants associated with beta-thalassemia: $\mathrm{Hb}$ Miami [beta 116 (G18)his-->Pro], and Hb Hershey [beta70(E14)Ala-->Gly], and a second unstable $\mathrm{Hb}$ variant at 170 : $\mathrm{Hb}$ Abington [beta70(E14)Ala->Pro]. Hemoglobin. 2005;29(4):241-8.

21. Grignoli CR, Wenning MR, Sonati MF et al. Hb Rio Claro [beta34(B16)Val-->Met]: a novel electrophoretically silent variant found in association with $\mathrm{Hb}$ Hasharon [alpha47(CE5)Asp-->His] and alpha-thalassemia-2(-alpha3.7). Hemoglobin. 1999;23(2): 177-82.

Avaliação: Editor e dois revisores externos

Conflito de interesse: não declarado

Recebido: 19/09/2007

Aceito: 25/09/2007 